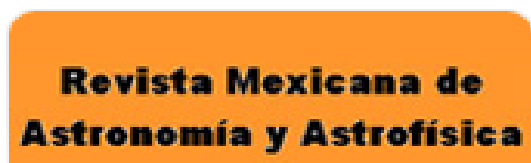
Astronomia y Astrofisica
Revista Mexicana de Astronomía y Astrofísica

ISSN: 0185-1101

rmaa@astro.unam.mx

Instituto de Astronomía

México

Allen, Christine; Pichardo, Bárbara; Moreno, Edmundo

SIX NEW GALACTIC ORBITS OF GLOBULAR CLUSTERS IN A MILKY-WAY-LIKE GALAXY

Revista Mexicana de Astronomía y Astrofísica, vol. 34, 2008, pp. 107-110

Instituto de Astronomía

Distrito Federal, México

Available in: http://www.redalyc.org/articulo.oa?id=57116170025

How to cite

Complete issue

- More information about this article

Journal's homepage in redalyc.org

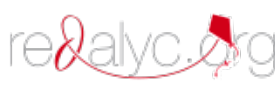

Scientific Information System 


\title{
SIX NEW GALACTIC ORBITS OF GLOBULAR CLUSTERS IN A MILKY-WAY-LIKE GALAXY
}

\author{
Christine Allen, ${ }^{1}$ Bárbara Pichardo, ${ }^{1}$ and Edmundo Moreno ${ }^{1}$ \\ RESUMEN
}

Recientemente se han publicado nuevos movimientos propios absolutos para seis cúmulos globulares. Esto nos ha motivado para obtener las órbitas galácticas de estos cúmulos en diferentes variantes del potencial galáctico. Hemos utilizado un modelo de potencial axisimétrico, uno con barra y otro con barra y brazos espirales. Comparamos y discutimos las características orbitales para esos tres casos.

\section{ABSTRACT}

Absolute proper motions for six new globular clusters have recently been published. This motivated us to obtain the Galactic orbits of these six clusters in several variants for the Galactic potential. We used an axisymmetric model, a barred potential and also a barred potential that includes spiral arms. The orbital characteristics are compared and discussed for these three cases.

Key Words: Galaxy: halo — Galaxy: kinematics and dynamics — globular clusters: general

\section{INTRODUCTION}

Absolute proper motions for six new globular clusters have recently been published (CasettiDinescu et al. 2007), thus increasing to 54 the number of globular clusters of our Galaxy for which full space velocities and Galactic orbits can now be calculated. In a previous paper (Allen, Moreno, \& Pichardo 2006) Galactic orbits were computed for 48 globular clusters, using both an axisymmetric and a barred Milky-Way-like potential. We found that the effect of the bar was greatest for clusters with orbits residing mostly within the bar, and negligible for the outermost clusters of our sample. Since most of the new clusters reside within the region of influence of the Galactic bar we have computed their orbits, in the axisymmetric potential of Allen \& Santillán (1991) and with the addition of the Galactic bar model produced by Pichardo, Martos, \& Moreno (2004) with moderate mass and length.

In particular, Casetti-Dinescu et al. (2007) comment on the apparent "pairing" of the orbital parameters of NGC 2808 and NGC 4372, on one hand and NGC 4833, and NGC 5986 on the other. Since these "pairings" or kinematic groups may have important implications for the dynamical and merger history of our Galaxy (Kepley et al 2007; Allen et al. 2007 and references therein), it is interesting to assess whether or not these similarities are sensitive

\footnotetext{
${ }^{1}$ Instituto de Astronomía, Universidad Nacional Autónoma de México, Apdo. Postal 70-264, 04510, México, D. F., Mexico (chris@astroscu.unam.mx).
}

to the Galactic model potential used, and how they are affected by the bar.

Since the permanence of bar-like structures in galaxies is a matter of debate, we cannot be sure that the bar of our Milky Way has existed throughout the Galactic lifetime. Therefore, we also inquire how the orbits would look like if the bar has existed for only about a third of this lifetime.

To investigate the possible effects of spiral structure on the orbital characteristics of the clusters, we also include computations of the six globular cluster orbits in a Galactic potential that incorporates spiral arms.

\section{GALACTIC POTENTIAL AND INITIAL CONDITIONS}

For our study we will employ the axisymmetric Galactic potential of Allen \& Santillán (1991), the barred Galactic potential of Pichardo et al. (2004), and the spiral-arms model of Pichardo et al. (2003). To model the spiral perturbation we proceeded as described in Allen, Moreno, \& Pichardo (2008).

The initial conditions were obtained using the absolute proper motions provided by Casetti-Dinescu et al. (2007). Other relevant data were taken from the compilation by Harris (1996). Once the space velocities are known, we integrated the orbits backwards in time for $1.5 \times 10^{10}$ years. For the orbital computations we used the Bulirsch-Stoer algorithm. In the axisymmetric case the relative errors in the total energy were of about $10^{-14}$ at the end of the integration. In the barred potential, the orbits are 


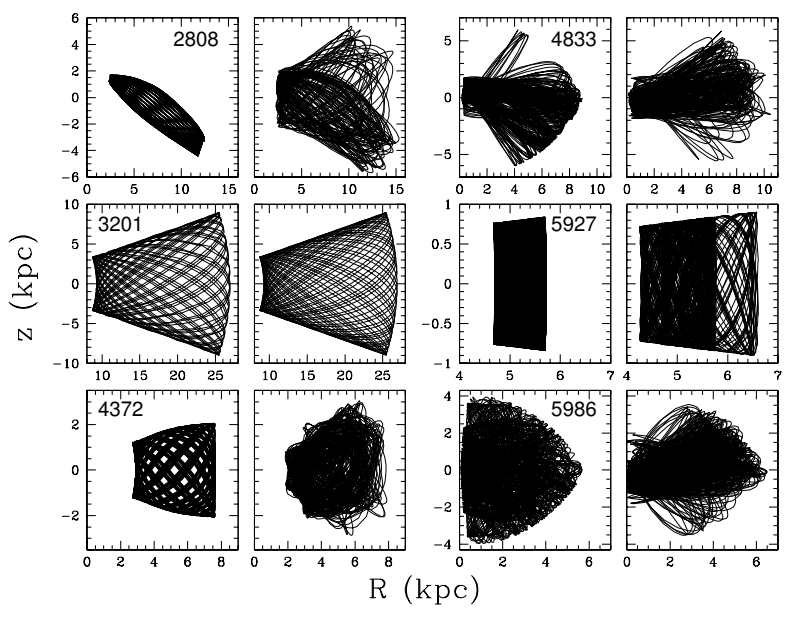

Fig. 1. Meridional Galactic orbits of the six clusters during the lifetime of the Galaxy. For each cluster, the orbits in the axisymmetric (left) and barred (right) potentials are shown.

computed in the non-inertial reference frame where the bar is at rest. In this case the precision of the calculations can be checked using Jacobi's constant. The relative errors in this quantity turn out to be, typically, $10^{-10}$ to $10^{-11}$.

\section{THE GALACTIC ORBITS}

Figure 1 shows the meridional orbits of the six clusters in both the axisymmetric and the barred potentials. The orbital parameters for both cases are given in Table 1 of Allen et al. (2008). To take into account the influence of observational uncertainties, we computed for each cluster two additional orbits in both the axisymmetric and barred potential. Initial conditions for these orbits were chosen so as to maximize and minimize the energy, in order to obtain two extreme orbits that bound observational errors. The orbital parameters for these extreme orbits are also given in Allen et al. (2008) for different model potentials.

In contrast to the expectations of CasettiDinescu et al. (2007), we find that five of the six new clusters are noticeably affected by the bar. The only exception is NGC 3201, the least bound of the clusters. This orbit always remains entirely outside the bar region.

As can be seen in Figure 1, the orbits of the five remaining clusters are significantly affected by the bar. Their orbital parameters show fairly large changes, reaching higher values of apogalactic distance and distance to the Galactic plane, as well as exhibiting noticeably more chaos.

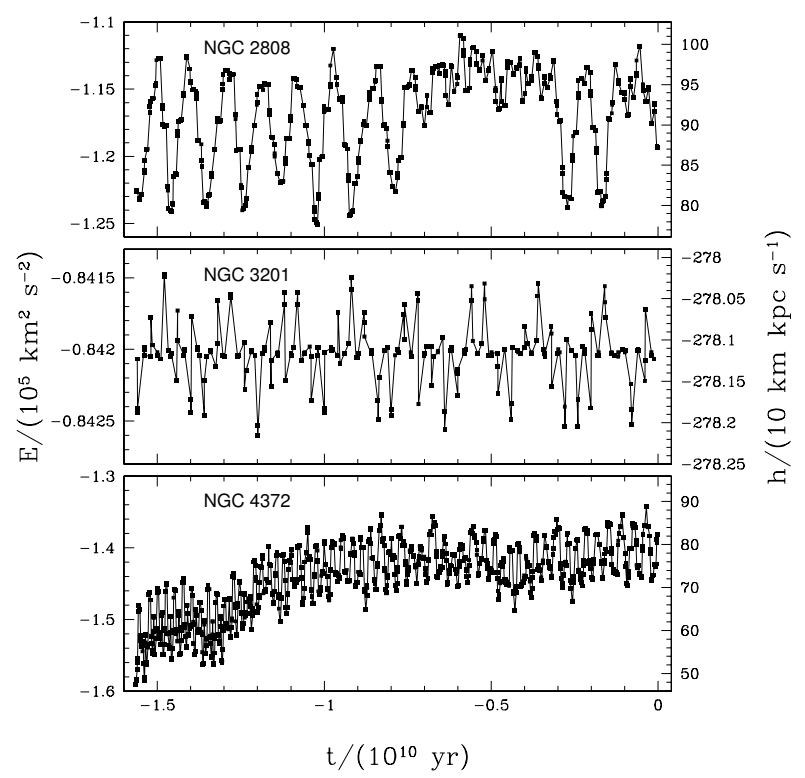

Fig. 2. Energy and $z$-angular momentum as functions of time in NGC 2808, NGC 3201, and NGC 4372, with the barred potential.

The orbits of NGC 4833 and NGC 5986 are chaotic both without and with the bar. The orbit of NGC 2808 is near-resonant in the axisymmetric case, but becomes more chaotic and reaches greater radii and $z$-distances in the presence of the bar.

NGC 5927, a thick disk cluster, has a tightly confined box-type orbit in the axisymmetric case. This orbit becomes larger in the presence of the bar, reaching radii of almost $7 \mathrm{kpc}$.

Finally, the orbit of NGC 4372, clearly quasiperiodic in the axisymmetric case, becomes chaotic with the bar, and reaches larger $z$-distances.

Plots of the run of energy, $E$, and $z$-component of angular momentum, $h$, were obtained for all clusters. They are shown in Figures 2 and 3. These quantities are, of course, conserved in the axisymmetric case. In the presence of the bar, the orbits generally do not show large secular changes in $E$ or $h$. Indeed, these quantities are conserved on the average within better than 10 percent. But periodic or quasi- periodic changes are seen to occur in all cases. In Figures 2 and 3 we can see that NGC 3201, and NGC 5927 show small periodic or quasi-periodic changes. NGC 5986 shows abrupt changes in the angular momentum that occasionally reverse the sense of revolution of the cluster around the Galactic center.

To obtain a rough idea of the changes in the orbits that would appear if the Galactic bar were not a permanent feature, we computed the orbits back- 


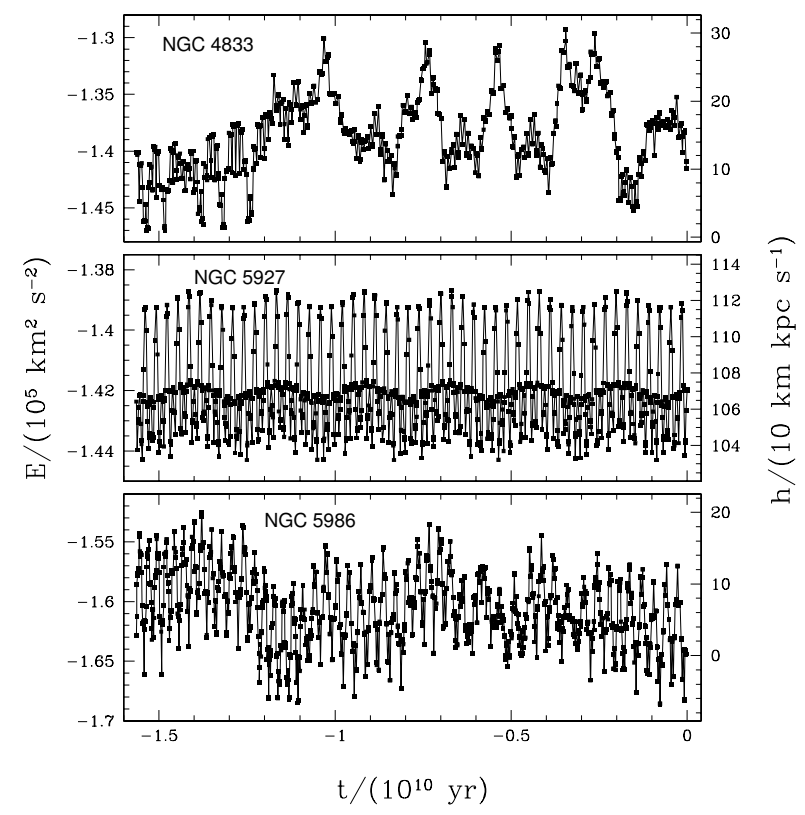

Fig. 3. As in Figure 2, for NGC 4833, NGC 5927, and NGC 5986.

ward in time, but only for the last 5.0 Gyr, that is, approximately a third of the Galactic lifetime. Figure 4 shows the orbits we obtained. A comparison of Figure 1 and Figure 4 clearly shows that the effects of the bar are similar for both cases. The sole exception is NGC 2808, for which more than 5 Gyr are necessary for the orbit to become non-resonant. We conclude that even if the Galactic bar has existed only for the last third of the Galactic lifetime, its effect on the orbits of the "inner" clusters is quite as noticeable as that of a bar present throughout the history of the Galaxy.

A comparison of the orbital parameters we obtain with those of Casetti-Dinescu et al. (2007) shows satisfactory overall agreement. In general, the uncertainties resulting from observational errors are larger than the ones resulting from using different axisymmetric Galactic potential models. However, our results on the orbits of the cluster "pairings" found by them (NGC 5986-NGC 4833 and NGC 2808-NGC 4372) are not similar enough to support such "pairings". The orbital parameters in the barred potential differ even more. So, we have to regard the "pairings" as not established by the orbital parameters we obtain here.

\section{THE EFFECTS OF SPIRAL ARM PERTURBATIONS}

To study possible effects of spiral structure on the orbital characteristics of the clusters we have com-

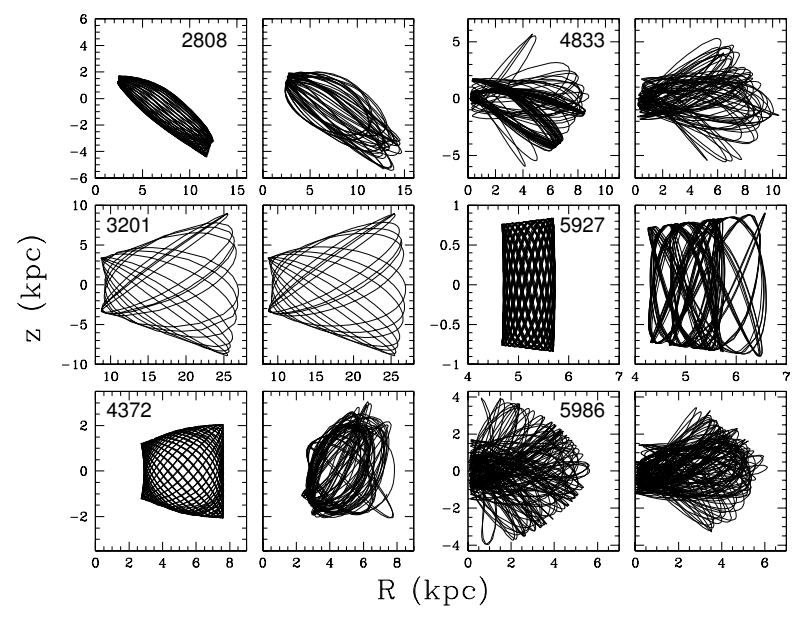

Fig. 4. As in Figure 1, now for the last 5 Gyr.

puted the six orbits using a Galactic potential that includes spiral arms. A priori, one would expect the effect of spiral structure to be small since the mass of the spiral features is small compared to that of the disk. Also, the great majority of the globular clusters spend most of their lifetimes away from the Galactic plane. However, this is not the case for the thick-disk globular clusters.

We integrated the six globular cluster orbits for the last 5 Gyr only, because this time span proved to be sufficient to show the effect of the spiral arms. The orbits were integrated in two variants of the potential, both including the axisymmetric part. In one variant we added the spiral arms, and in the other, the spiral arms and the bar. In Figure 5 we show the results of the computations in the form of meridional plots.

If we compare the orbits in the pure axisymmetric potential (Figure 1) with those in the potential with spiral arms we can clearly see the effect of the latter. The orbits change their shape in all cases, although only for clusters remaining closest to the Galactic plane (such as NGC 5927, NGC 4372, NGC 2808) the effect is noticeable. Again, the least affected orbits are those that reach large radial and vertical distances and remain mostly far from the influence of the arms, as is the case of NGC 3201.

The effect of the spiral perturbation is most pronounced in the case of near- resonant (like that of NGC 2808), or irregular orbits (like NGC 4833 and NGC 5986). Another unexpected result is seen in the orbit of NGC 4833, which is more symmetrical with respect to the Galactic plane in the presence of the spiral perturbation. 


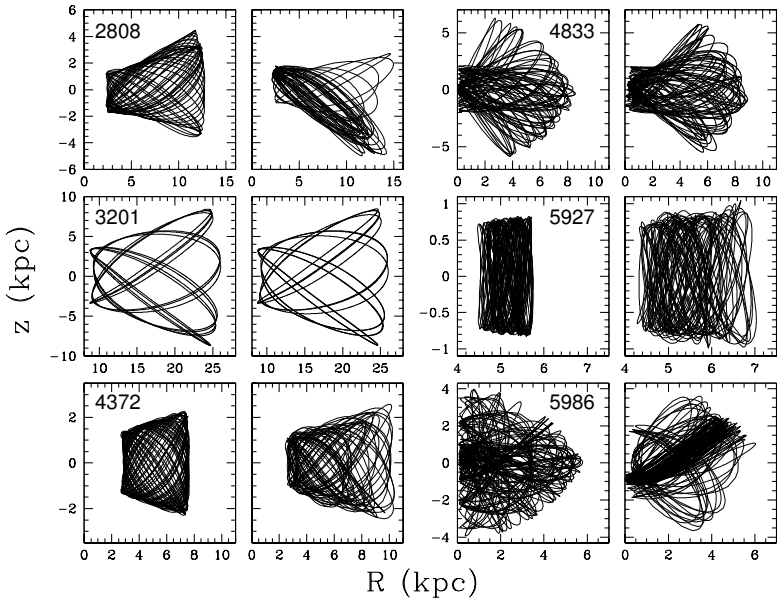

Fig. 5. The effect of the spiral arms. Meridional Galactic orbits of the six clusters during the last 5 Gyr. For each cluster, the left frame shows the orbit in the axisymmetric plus the two-spiral-arm potential, and the right frame shows the orbit in the barred (axisymmetric + bar) plus the two-spiral-arm potential.

The six orbits here studied show little change in the orbital parameters (such as the maximum absolute values of the $z$ - distances and the peri- and apogalactic distances) due to the presence of spiral arms. The cluster orbits are generally diffused outwards. This effect is largest for clusters remaining close to the Galactic plane $(z \lesssim 2 \mathrm{kpc})$, and inside the radial zone of strong influence of the spiral arms $(3<R<8 \mathrm{kpc})$.

Even though, in general, the dynamical effects of the bar dominate over those of the spiral arms, it is interesting to see that the spiral perturbations may play a non-negligible role. The clearest example is that of NGC 2808.

\section{DISCUSSION AND CONCLUSIONS}

We have obtained orbits for 6 additional globular clusters in both a barred and an axisymmetric Galactic potential, as well as in a potential including spiral arms. The total number of clusters with available orbits is now 54. Among the newly calculated cases, only the orbit of NGC 3201, an "outer" cluster, is unaffected by the bar. We find that the main changes the bar causes in the orbits are larger vertical and radial excursions, and far more chaos. In general, the bar causes no net global changes in the energy or the $z$-component of the angular momentum. However, in four out of the six cases, jumps in these quantities do occur, even causing a temporary reversal of the sense of rotation of the orbit. The effect of a shorter-lived bar is found to be quite as noticeable on these orbits as that of the longer-lived one.

Orbits with spiral-arm perturbations were also computed. Contrary to expectations, even a small perturbation, accounting for only $5 \%$ of the local arm-to-disk radial force ratio, causes changes in the form of the orbits. Although the influence of the spiral arms on the orbit of the clusters closest to the Galactic disk is not negligible, the long term effects are definitely dominated by the Galactic bar.

\section{REFERENCES}

Allen, C., Moreno, E., \& Pichardo, B. 2006, ApJ, 652, 1150

$$
\text { 2008, ApJ, 674, } 237
$$

Allen, C., Poveda, A., \& Hernández-Alcántara, A. 2007, in IAU Symp. 240, Binary Stars as Critical Tools \& Tests in Contemporary Astrophysics, ed. W. I. Hartkopf, E. F. Guinan, \& P. Harmanec (Cambridge: Cambridge Univ. Press), 405

Allen, C., \& Santillán, A. 1991, RevMexAA, 22, 255

Casetti-Dinescu, D. I., Girard, T. M., Herrera, D., van Altena, W. F., López, C. E., \& Castillo, D. J. 2007, AJ, 134, 195

Harris, W. E. 1996, AJ, 112, 1487

Kepley, A. A., et al. 2007, AJ, 134, 1579

Pichardo, B., Martos, M., \& Moreno, E. 2004, ApJ, 609, 144

Pichardo, B., Martos, M., Moreno, E., \& Espresate, J. 2003, ApJ, 582, 230 